\title{
Measurement of motility of Helicobacter pylori, Campylobacter jejuni, and Escherichia coli by real time computer tracking using the Hobson BacTracker
}

\author{
Q N Karim, R P H Logan, J Puels, A Karnholz, M L Worku
}

\begin{abstract}
Aims-(1) To make precise measurements and comparisons of various aspects of motility of three gastrointestinal pathogens, Helicobacter pylori, Campylobacter jejuni, and Escherichia coli, in log phase growth; (2) to provide background information on motility data to study the influence of $\mathrm{pH}$, viscosity, and chemotactic factors, thereby gaining a better understanding of bacterial pathogenesis.

Methods-Computer image processing technology and phase contrast microscopy (Hobson BacTracker) were used to measure several indices of bacterial motility in real time. Ten clinical isolates of each species in log phase liquid culture were studied.

Results-C jejuni moved fastest, with a median curvilinear velocity (CLV) of 38.76 $\mu \mathrm{m} / \mathrm{s}$ (range 29.08 to 52.82 ). Next was $H$ pylori, median CLV $25.02 \mu \mathrm{m} / \mathrm{s}$ (range 12.07 to 29.07). $E$ coli was the slowest, median CLV $12.73 \mu \mathrm{m} / \mathrm{s}$ (range 8.20 to 18.04). The straight line velocities showed similar trends. Measurement of track linearity (TL) showed that $C$ jejuni moved the straightest (TL 60.3\%), $H$ pylori moved in wide circles (TL $28.7 \%$ ), and $E$ coli showed spinning movement without much linear displacement (TL 18.3\%). There were significant differences in these three variables between the species studied, but no significant differences in measurements of time and frequency of halts between movement runs.

Conclusions-The BacTracker provides a useful technical aid for measuring several indices of bacterial motility objectively, reproducibly, and precisely, which is difficult to achieve without computer assistance. Accurate quantification of motility provides a basis for studying the factors which influence bacterial motility. It can provide phenotypic measurements of the effect of flagellar gene depletion. (F Clin Pathol 1998;51:623-628)
\end{abstract}

Keywords: bacterial motility measurement; computer image processing technology; bacterial tracking

For many bacteria, motility confers a survival advantage by permitting migration towards a favourable microenvironment, or away from an unfavourable one. The operation of motility machinery is very energy expensive for micro- organisms, and the slightly slower growth of motile flagellate cells would ensure the loss of the motile phenotype if it did not confer some substantial benefit to the cell, as explained by Armitage. ${ }^{1}$ Microbial motility can be assessed microscopically by hanging drop, by phase contrast and dark ground illumination, or macroscopically, by growth in semisolid agar, swarming on surface of solid agar, or by the crossing of a filter paper bridge over a trench cut in solid agar.

These methods distinguish motile from nonmotile bacteria and assess motility only subjectively, though giving the impression that some bacteria move faster than others. The methods are not precise enough to follow transient or minor changes in motility. Video recording of motile bacteria has provided a means of quantifying motility. The video recorded tapes have been replayed, and motility measured manually on the screen by planimeter and stop watch, as described by Ferrero and Lee. ${ }^{2}$ Such manual measurements are cumbersome and difficult to do on large numbers of bacteria.

These problems can be overcome by the Hobson BacTracker (Hobson Tracking System, Sheffield, UK), which allows precise and objective measurements of motility. It is a measuring system that incorporates unique "blob and track" image processing technology, analysing bacterial movement in real time. The tracker comprises a phase contrast microscope connected to a video camera. The camera is linked to a video recorder, which is connected to a computer that tracks 120 moving bacteria on screen simultaneously and continuously. The results are displayed as histograms or trail draws. This system records several indices of motility including direction, curvature rates, curvilinear velocity, and straight line velocity, which can be measured accurately, objectively, and reproducibly so that comparisons can be made under different experimental conditions. Recordings of movement can be stored in the computer and can be downloaded for more detailed statistical analysis.

Real time computer tracking has been used to assess the motility of Rhodobacter sphaeroides, Rhodospirullum rubrum, and Salmonella typhimurium. ${ }^{3}$ The aim of our present study was to assess the motility of Helicobacter pylori ( $H$ pylori), Campylobacter jejuni ( $C$ jejuni), and Escherichia coli $(E$ coli). These are major pathogens infecting the upper and lower gastrointestinal tract, in which motility is acknowledged to be a virulence factor. ${ }^{24-6}$ Motility measure- 


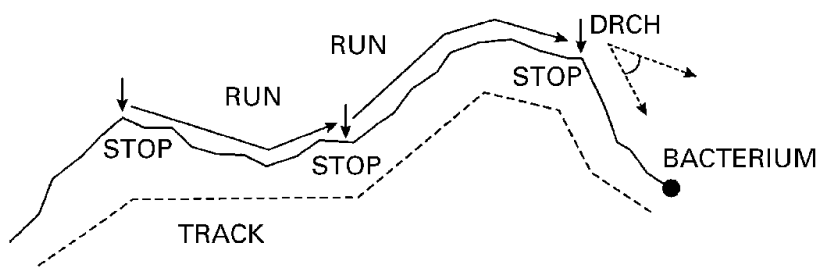

A TRACK is the path travelled by a moving cell A RUN is a length of TRACK between two STOPS $\mathrm{DRCH}-$ Direction changed in degrees

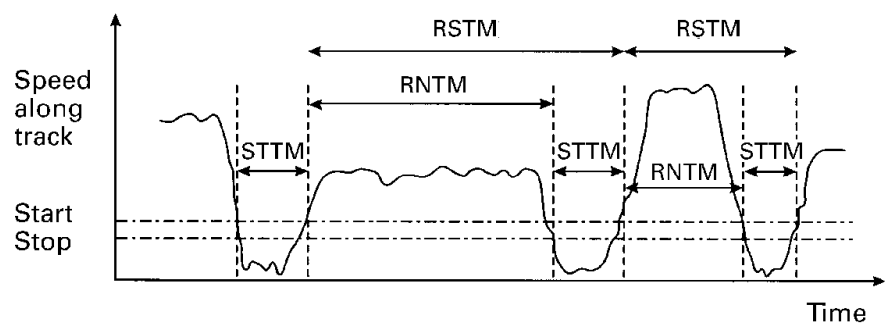

STTM - Stop time, RNTM - Run time, RSTM - Run stop time

Figure 1 Quantities analysed by the BacTracker.

ments of these bacteria in log phase growth can form the basis for studying and comparing changes that take place because of different environmental factors, such as phases of growth, $\mathrm{pH}$, temperature, and the presence of antimicrobial agents. These measurements can be used to study chemotaxis and help us to understand this aspect of bacterial physiology and pathogenesis.

\section{Methods}

THE HOBSON BACTRACKER

The BacTracker separates the moving parts of the image from the static parts. The moving object is then filtered and a threshold imposed to produce coloured "blobs" which are superimposed on the moving objects on the black and white video image. Once the moving objects have been identified the BacTracker has the coordinates of every moving object in the frame and with this information various measurements can be made. The BacTracker is a real time image processing system where motility measurements are carried out as they are occurring - the computer measures continuously and does not have to stop, calculate, and measure as in snapshot measurements made over a short period of time. Various measurements are displayed on the computer screen, such as summary graphs and histograms, while the tracking screen shows the cells as they are tracked. Thus the system can be validated while watching in real time. The video images are processed at $25 \mathrm{~Hz}$ in PAL format, or $30 \mathrm{~Hz}$ or $6 \mathrm{~Hz}$ in NTSC North American format.

VARIABLES MEASURED

The following variables were measured by the BacTracker:

Track-A track is the path travelled by a moving cell. It is measured from the point of detection by the computer until the cell disap- pears from view or moves out of the analysis window (fig 1).

Stop-A stop occurs when the speed of the bacterial cell falls below the stop speed defined in the setting screen. This was set at the speed shown by Brownian movement of dead bacteria.

Run-A run is the track between two stops (fig 1).

Curvilinear velocity (CLV)-This is the length of a track divided by the time taken to travel it. It is calculated by summing the incremental distances moved in each frame along the sampled path and divided by the total time for the track. It is measured for tracks (total path length) and for runs (incremental path lengths between two stops) in $\mu \mathrm{m} / \mathrm{s}$ (fig 2 ).

Straight line velocity (SLV)-This is calculated by measuring the straight line distance between the start and end point of the track and dividing by the time taken to travel it. It is measured in $\mu \mathrm{m} / \mathrm{s}$ (fig 2 ).

Track linearity percentage (TL\%)-This is the ratio of the straight line velocity to curvilinear velocity $\times 100(S L V / C L V(100))$. For a bacterium that runs straight this value is $100 \%$. The more curved the route taken by the bacterium the greater will be the curvilinear velocity, and the lower the straight line velocity and the value of track linearity percentage. For a bacterium that spins around a point the straight line velocity may be so small compared with the curvilinear velocity that the value may approach $0 \%$. Thus, depending on the straightness or curvature of the path of the bacterium, the value of track linearity will be between $100 \%$ and $0 \%$. This is shown diagrammatically in fig 2 .

Curvature rate (CVRT॰/s)-This is measured using the incremental sum of change in angle as the bacterium changes direction for the length of the track. It includes the sign to reflect the direction of change. For each track point the signed change in angle from the previous track point is measured. The sum of the signed change of angle is accumulated along the track. At the end of the track the sum of the signed angle change is divided by the time for the track to give a value for the curvature rate in degrees per second. For bacteria with net anticlockwise movement the value is positive, and for a bacteria with net clockwise the value is negative. This is shown as direction change (DRCH) (fig 1).

Stop time (STTM)-This is the time of a defined stop between two adjacent runs. The average of all the stop time is displayed in seconds (fig 1).

Stop frequency (STFRQ)-This is a measure of how often the cell stops. The time is measured from the start of a run through to the end of the following stop or the start of a new run. This time is divided into 1 to give a frequency in $\mathrm{Hz}$ or times/s. A value is displayed as an average of each track.

Limits - When bacteria travel at a varying velocity, the BacTracker measures the median velocity and the histogram shows the distribution of bacteria with different velocities. By setting the "limits" values to a high or low 
velocity one can include or exclude the fast or slow moving bacteria and preferentially study the fast or the slow bacteria in a mixed population.

ISOLATION OF BACTERIA

We used 10 isolates of each species of $E$ coli, $H$ pylori, and $C$ jejuni, obtained from specimens received for diagnostic purposes. Reference strains $H$ pylori NCTC $11637, E$ coli NCTC 10413, and a clinical isolate of $C$ jejuni used as a laboratory control were also included. The $E$ coli strains were isolated from patients with bacteraemia or urinary tract infection. They were identified by standard laboratory procedures using the API $20 \mathrm{E}$ system (API, Lyon, France). The $C$ jejuni were isolated by culturing stool on Skirrow's media ${ }^{7}$ at $42^{\circ} \mathrm{C}$ for 24 hours in a

A

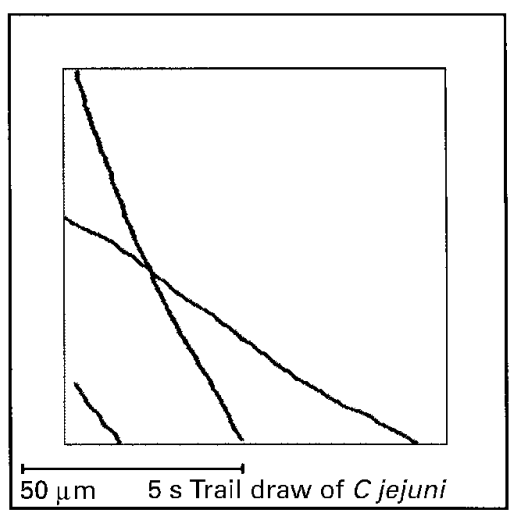

B

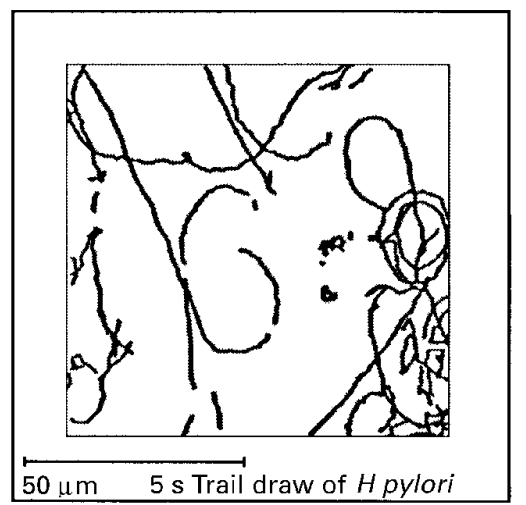

C

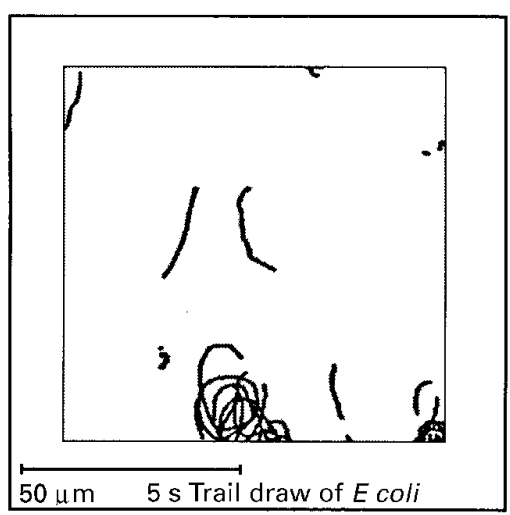

microaerophilic environment using gas packs (Campypak, BBL, Maryland, USA) in gas jars (Oxoid, Basingstoke, UK). The colonies were identified by typical appearance on Gram film and oxidase test. The $H$ pylori were isolated by plating the antral biopsies on Columbia agar (BBL) with $7 \%$ horse blood (TSCL, Buckingham, UK) made selective by adding antibiotic supplements of $20 \mathrm{mg} / \mathrm{l}$ nalidixic acid, $2 \mathrm{mg} / 1$ amphotericin $\mathrm{B}$, and $3 \mathrm{mg} / \mathrm{l}$ vancomycin. The plates were incubated for 72 hours at $37^{\circ} \mathrm{C}$ in a microaerophilic environment using Campypaks as above. Only one subculture was made from plates that showed heavy confluent growth on primary culture. This subculture was incubated for 48 hours as above and used for motility studies. Only the first subculture was used for this study. This was to avoid repeated subcul-
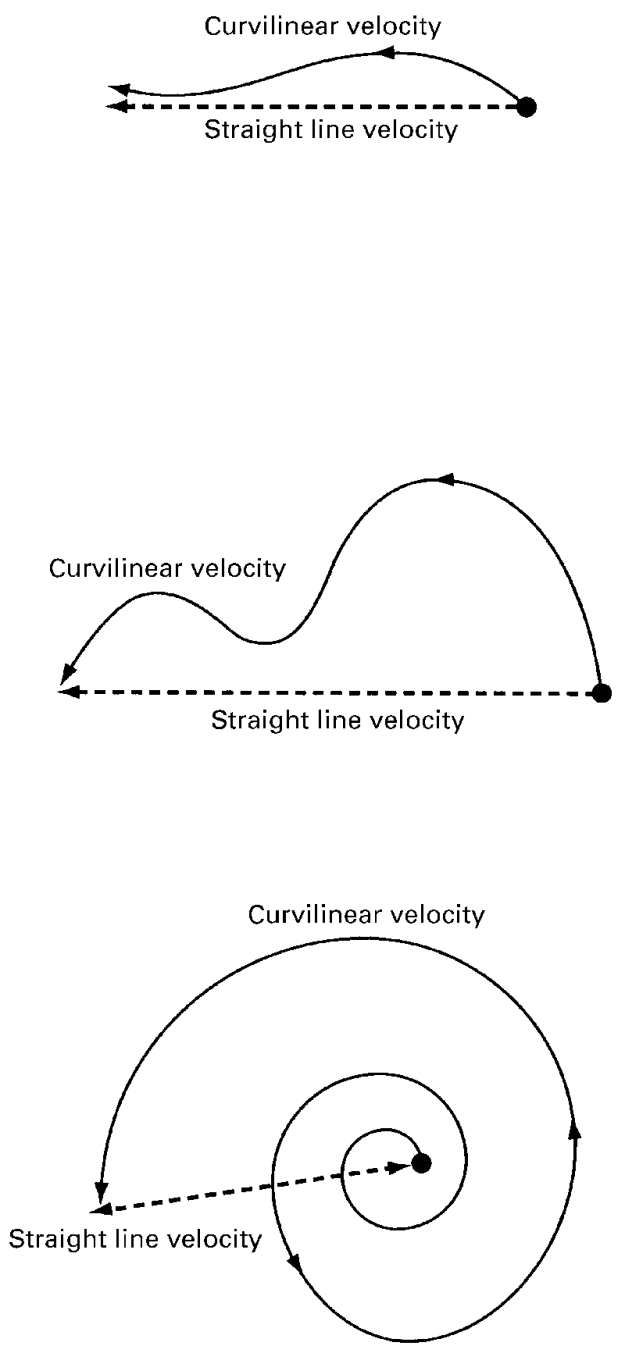

Track linearity percentage $(T L \%)=\frac{\text { SLV }}{C L V} \times 100$

Figure 2 Left panels: computer tracking of 5 second trail draws in real time of $(A) C$ jejuni, (B) H pylori, and (C) E coli. Right panels: Diagrammatic representation of relation between straight line velocity (SLV), curvilinear velocity (CLV), and track linearity (TL\%). Track linearity values: (A) $83 \%$; (B) 44\%; (C) $14 \%$. 
Table 1 Comparison of different indices of mobility of $E$ coli, $H$ pylori, and $C$ jejuni. Ten isolates were examined for each species

\begin{tabular}{|c|c|c|c|c|c|}
\hline Measurement & E coli & ( $p$ Value) & H pylori & ( $p$ Value) & C jejuni \\
\hline Curvilinear velocity $(\mu \mathrm{m} / \mathrm{s})$ & $12.73(8.20$ to 18.04$)$ & 0.0036 & $25.02(12.47$ to 29.07$)$ & 0.0008 & $38.76(29.08$ to 52.82$)$ \\
\hline Straight line velocity $(\mu \mathrm{m} / \mathrm{s})$ & $2.27(0.16$ to 9.15$)$ & 0.0113 & $7.2(1.05$ to 14.31$)$ & 0.0022 & $23.41(8.00$ to 26.00$)$ \\
\hline Track linearity (\%) & 18.3 & 0.0173 & 28.7 & 0.0376 & 60.3 \\
\hline Curvature rate $(\% / s)$ & +117.8 & 0.1212 & +157.2 & 0.0140 & +251.2 \\
\hline Stop frequency/s $(\mathrm{Hz})$ & $1.05(0.29$ to 1.33$)$ & 0.0890 & $1.25(0.82$ to 1.61$)$ & 0.0890 & $1.07(0.89$ to 1.27$)$ \\
\hline Stop time (s) & $0.44(0.29$ to 0.65$)$ & 0.256 & $0.67(0.26$ to 0.53$)$ & 0.9097 & $3.05(0.24$ to 0.41$)$ \\
\hline
\end{tabular}

Values are medians (range).

tures as many strains of $H$ pylori became coccoid and non-motile after three to four subcultures.

BACTERIAL PREPARATION

Growth curves were determined in liquid cultures to find the inoculum to be used and the period of incubation required to attain the log phase of growth. Our preliminary experiments showed that $H$ pylori moved fastest in their log phase, ${ }^{8}$ although this may not be the case with $E$ coli, as explained in the discussion. $E$ coli and $C$ jejuni were inoculated in peptone water, and $H$ pylori in brain-heart infusion (BHI) broth enriched with newborn calf serum and the antibiotic supplements detailed above. The peptone water inoculated with $C$ jejuni and BHI broth inoculated with $H$ pylori were distributed in 5 $\mathrm{ml}$ volumes in $60 \mathrm{ml}$ plastic flat bottomed screw capped containers. The caps were loosened and the cultures incubated in an upright position without agitation in an atmosphere of $8 \% \mathrm{CO}_{2}$ in air at $37^{\circ} \mathrm{C}$ for 48 hours for $C$ jejuni and $H$ pylori. The peptone water inoculated with $E$ coli was incubated overnight at $37^{\circ} \mathrm{C}$ in ordinary atmosphere. Immediately after inoculation and at timed intervals, optical densities and viable counts were performed for each of the isolates for the period of incubation. All the optical densities were measured at $540 \mathrm{~nm}$.

Plate cultures of the respective bacteria were used for inoculating bacterial suspensions for motility measurements. This was to ensure purity of culture at the point of inoculation to the liquid media. The dilutions of the bacterial suspensions were adjusted on the basis of their optical densities to correspond to $10^{6}$ colony forming units $(\mathrm{CFU}) / \mathrm{ml}$, as determined by the growth curve experiments; $500 \mu \mathrm{l}$ of this inoculum were added to $5 \mathrm{ml}$ of the corresponding liquid cultures and incubated as described above. Motility was measured after three hours for $E$ coli and after 24 hours for $C$ jejuni and $H$ pylori, when they had attained the logarithmic phase following static incubation. This was confirmed by measuring serial optical density values and viable counts at timed intervals during this incubation.

MICROSCOPY

Each of the liquid cultures was drawn into rectangular capillary microslides of internal diam- eter of $100 \mu \mathrm{m}$ (Camlab VD/5010 050, Cambridge, UK) and observed under phase contrast microscope at $40 \times$ magnification on a stage slide heater (MS100 Linkam Scientific Instruments, Surrey, UK) at $37^{\circ} \mathrm{C}$. The ends of the slides were sealed with vinyl plastic putty to avoid drifts during measurements of motility. The BacTracker was set to measure 100 tracks for each bacterium, and calibrated for $40 \times$ magnification for the motility measurements. Brownian movements were excluded by tracking and measuring formalin killed bacteria; this corresponded to a curvilinear velocity of up to $5.0 \mu \mathrm{m} / \mathrm{s}$ and a straight line velocity of up to 2.0 $\mu \mathrm{m} / \mathrm{s}$.

\section{STATISTICS}

The results were analysed by the Minitab statistical package. The distributions of the various indices of motility were nonparametric, so the Mann-Whitney U test was used to determine statistical significance.

\section{Results}

Of the three species studied, $C$ jejuni moved fastest with a median curvilinear velocity of $38.76 \mu \mathrm{m} / \mathrm{s}, H$ pylori were next with a median of $25.02 \mu \mathrm{m} / \mathrm{s}$, and $E$ coli were slowest with a median of $12.37 \mu \mathrm{m} / \mathrm{s}$ (table 1 ). Using the Mann-Whitney $U$ test, the differences in motility were statistically significant between $E$ coli, $H$ pylori, and $C$ jejuni. The straight line velocity also showed the same ranking, the median values being $23.41 \mu \mathrm{m} / \mathrm{s}$ for $C$ jejuni, $7.20 \mu \mathrm{m} / \mathrm{s}$ for $H$ pylori, and $2.27 \mu \mathrm{m} / \mathrm{s}$ for $E$ coli. These differences were statistically significant between the three species (table 1).

Assessment of tracking linearity showed that $C$ jejuni moved the straightest, with the highest TL value of $60.3 \%$. H pylori moved in wide circles with a TL value of $28.7 \%$, and $E$ coli had a spinning movement, with the lowest value TL value $(18.3 \%)$. This is shown in their representative trail draws (fig 2).

The comparative values and distribution of curvilinear velocity, straight line velocity, and tracking linearity of the individual strains of the three genera are shown in fig 3. The corresponding curvature rate, reflecting the signed change in angle, was $+117.8^{\circ}$ for $E$ coli, $+157.2^{\circ}$ for $H$ pylori, and $+251.2^{\circ}$ for $C$ jejuni. 

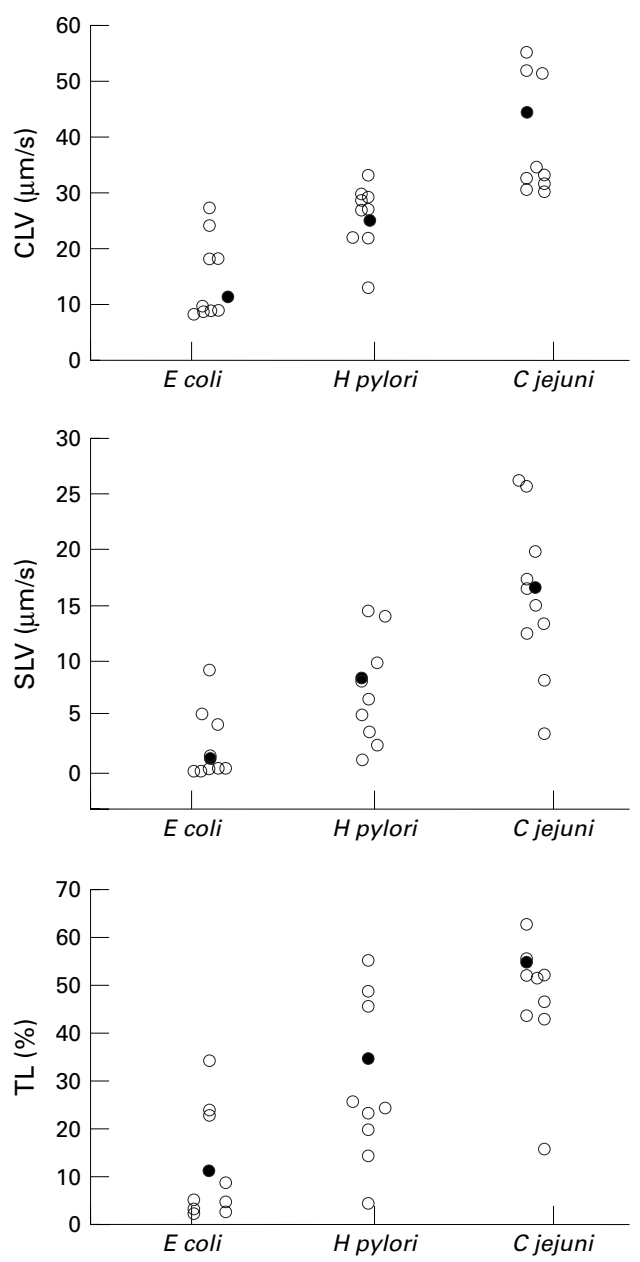

Figure 3 Distribution of the median values of curvilinear velocity (CLV), straight line velocity (SLV), and track linearity (TL) for each of the 10 strains of $E$ coli, $H$ pylori, and $C$ jejuni. Filled circles are reference strains.

The difference between $H$ pylori and $C$ jejuni was statistically significant but not the difference between $E$ coli and $H$ pylori (table 1 ). The overall movement of all three bacterial species was positive or anticlockwise.

The median stop frequency was similar for the three groups, being 1.05 for $E$ coli, 1.25 for $H$ pylori, and 1.07 for $C$ jejuni; the differences in these values were not statistically significant between the three species. The median stop times for the corresponding groups were 0.44 seconds for $E$ coli, 0.67 seconds for $H$ pylori, and 3.05 seconds for $C$ jejuni. There was no statistical difference in these variables between the three species (table 1).

\section{Discussion}

Accurate measurements are an important requirement for studying bacterial motility. Only with accurate measurements can transient or subtle changes in motility under the influence of chemicals and varied growth conditions be studied. These factors warrant further investigation, and the convenience of quantifying motility should aid such studies. Precise measurements are also needed for measuring bacterial chemotaxis. Bacteria vary in their motility in different phases of growth. ${ }^{8}$ Among the 10 strains of each species studied, $E$ coli showed the most strain variation in motility measurements. Such strain variation was observed less with $H$ pylori, which was more affected by the phase of growth. $C$ jejuni showed the least strain variation as far as motility was concerned. The relevance of these variations in terms of bacterial physiology and disease potential has to be explored. The motility of each of the three species was studied in one medium only; further studies in different media are required to examine the influence of media constituents on bacterial motility.

The Hobson BacTracker allows precise, objective measurements of motility, where the data can be stored and analysed. Its particular advantage is its ability to study large numbers of bacteria in a very short time, which facilitates statistical analysis. In a typical sample it is possible to measure 1000 bacteria in approximately 40 seconds. ${ }^{9}$ The technique is not prone to subjective errors, is reproducible, and allows many different variables to be studied simultaneously. This is not possible without computer aid, which makes a valuable contribution to the measurement of bacterial motility. However, the benefit of these various measurements needs to be evaluated and not all may be relevant to a particular set of experiments. The limitations are that, although individual bacteria are tracked, in any population the final result is a mean or a median value of all the bacteria tracked. This is therefore dominated by the measurements contributed by the majority of the population. and one has to depend on the histograms to see the distribution of individual measurements of the bacterium in relation to the whole population. However, one can selectively study the faster or slower bacteria by presetting the "limits" of individual measurements. To study a single bacterium, then, the specimen has to be so diluted that only one bacterium is visible in the field.

The ease of measuring bacterial motility enabled us to study the comparative motility of these three genera that infect the gastrointestinal tract. We saw different behaviour in the movement of these organisms in their log phase of growth in liquid culture. From conventional motility studies we know that bacteria are motile, and routine microscopy gives the impression that some are more motile than others, but the impression is subjective and cannot easily be quantified. We observed and quantified differences in the motility of the tree bacterial species, $C$ jejuni being much faster than $H$ pylori, which in turn are faster than $E$ coli. The measurements were performed in the log phase, where many bacteria move quickly. This is supported by published work showing that in general the best flagellation occurs in the logarithmic phase, ${ }^{10}$ and that $H$ pylori produces 20 times more flagellar protein in the logarithmic phase. ${ }^{11}$ However, this is not the case for $E$ coli, which is known to control expression of flagella in part by catabolite repression, and motility is generally lower in the logarithmic phase. This may be the explanation of the observation in our experiment that $C$ jejuni and $H$ pylori moved much faster in the logarithmic phase than $E$ coli. Fur- 
ther studies are required to establish the relation of motility with growth phase in other bacterial species. $E$ coli also showed more spinning movements than translational movements, whereas the $H$ pylori generally moved in wide circles and $C$ jejuni movement was straighter.

We do not know the significance of these different patterns of movements. Since subtle changes can be measured continuously over a long period, the technique described should be useful in studying chemotaxis. It will also provide a phenotypic basis for the study of mutants where the flagellar genes have been partially or completely deleted, and the effect quantified in terms of alteration of motility. We have observed lack of motility of aflagellate mutants of proteus species compared with the actively motile wild type in previous experiments. Our preliminary studies have shown that, while different genera show similar changes in motility on exposure to varying $\mathrm{pH}$, their motility patterns vary widely in the face of altered viscosity and in the different phases of growth. We are carrying out further work on these aspects. Some species showed greater strain variation, which could have clinical significance. These bacteria need to be studied under different experimental conditions to evaluate the significance of their varied motility in terms of their physiology and pathogenesis.
This study was supported by grants received from the University of London and Abbott laboratories. We are indebted to Professor $G$ Hobson and $H$ Roe for their help in setting up the BacTracker program, to Dawn Beaumont who helped with the statistics, to $\mathrm{Mr}$ Jon White for medical illustrations, and to Dr J H Baron and Dr J J Misiwicz for their constant support encouragement and for providing us with clinical material.

1 Armitage J P. Behavioural responses in bacteria. Annu Rev Physiol 1992;54:638-714.

2 Ferrero RL, Lee A. Motility of Campylobacter jejuni in a viscous environment: comparison with conventional rodshaped bacteria. F Gen Microbiol 1988;134:53-9.

3 Poole PS, Sinclair DR, Armitage JP. Real time computer tracking of free-swimming and tethered rotating cells. Anal Biochem 1988;175:52-8.

4 Eaton KA, Morgan DR, Krakowa S. Motility as a factor in the colonisation of gnotobiotic piglets by Helicobacter pylori. J Med Microbiol 1992;37:123-7.

5 Eaton KA, Morgan DR, Krakowa S. Campylobacter pylori virulence factors in gnotobiotic piglets. Infect Immun 1989; 57:1119-25.

6 Burke DA, Axon AT. Adhesive E coli in inflammatory bowel disease and infective diarrhoea. BM7 1998;297:102-4.

7 Skirrow's campylobacter media. In: Mackie and McCartney, eds. Practical medical microbiology, 14th ed. Edinburgh: Churchill Livingstone, 1996:444.

8 Mandelstam J, McQuillen K, Dawes I, eds. The biochemistry of bacterial growth, 3rd ed, section 2: Cells and populations. Oxford: Blackwell Scientific Publications, 0000:15-18.

9 Karim QN, Ashton L, Sidebotham RL, et al. Measurement of Helicobacter pylori motility in different growth phases in conventional broth cultures by real time computer tracking [abstr]. F Med Microbiol 1996;44:X.

10 Kodaka H, Armfield AY, Lombard GL, et al. Practical procedure for demonstrating bacterial flagella. $\mathcal{F}$ Clin Microbiol 1982;16:948-52.

11 Josnhans C, Labigne A, Suerbaum S. Reporter gene analysis shows that expression of both $\mathrm{H}$ pylori flagellins is dependent upon the growth phase [abstr]. Gut 1995; 37(suppl 1):264. 